\title{
Possible Striking Signals for a Quark-Gluon Plasma at RHIC
}

\author{
S.J. Lindenbaum ${ }^{a, b}$ and R.S. Longacre ${ }^{a}$ \\ ${ }^{a}$ Brookhaven National Laboratory, Upton, NY 11973, USA \\ ${ }^{b}$ City College of New York, NY 10031, USA中
}

\begin{abstract}
We believe that one can have serious reservations as to whether heavy ion collisions (e.g. $100 \mathrm{GeV} / \mathrm{n} \mathrm{Au}+100 \mathrm{GeV} / \mathrm{n} \mathrm{Au}$ ) can lead to Thermal and Chemical equilibrium over large regions (particularly if it is assumed this happens whenever QGP is produced at RHIC-that is if it is produced).

It is at present not clear that the collision dynamics and times available will lead to this. An alternate scenario proposed by Van Hove where localized in rapidity bubbles of plasma may well be more probable, and may well occur at least some of the time, and some of the time mainly survive to the final state. If this occurs we have developed a series of event generators to extend and describe these phenomena. A Van Hove type $[6,7]$ spherical bubble at $\eta=0$ is embedded in a resonable event generator in qualitative agreement with Hijing etc[12]. The plasma bubble hadronized at a temperature of $170 \mathrm{Mev}$ according to the model developed by Koch, Müller and Rafelski[21]. The amount of available energy assumed in the bubble is selected by that in a small central circular cross-section of radius $\approx 1.3 \mathrm{fm}$ or $\approx 2.5 \mathrm{fm}$ in $100 \mathrm{Gev} / \mathrm{n} \mathrm{Au}+\mathrm{AU}$, central events. The results predict (with the assumptions stated, possible) Striking Signals which may allow strong evidence for a QGP which cannot be explained by alternative conventional physics arguments, and thus may be crucial elements in establishing a QGP. We are also applying these techniques to investigating Kharzeev and Pisarski bubbles of metastable vacua with odd CP.
\end{abstract}

\section{Introduction}

For about two decades there has been considerable interest in the possibility that at sufficiently high temperatures or baryon density one or more phase transitions will lead to formation of a quark-gluon plasma characterized by deconfinement, and perhaps chiral symmetry restoration $[1,2]$ Very high energy heavy ion collisions was the community consensus as the best hope of forming a quark- gluon plasma QGP and this led to RHIC [3] with its up to $100 \mathrm{GeV} /$ nucleon Au colliding with $100 \mathrm{GeV} /$ nucleon Au colliding beam accelerator.

RHIC is expected to possibly begin to provide experimental data in 2000. Therefore it is timely to ask the question what could some striking signals possibly be generated and detected which would provide very strong evidence, hopefully convincing, for a QGP.

\footnotetext{
${ }^{1}$ This research was supported by the U.S. Department of Energy under Contract No. DE-AC0298CH10886 and the City College of New York Physics Department
} 
Of course it may be that QCD is not correct in this new energy domain, or that lattice gauge calculations and phenomenological models used to predict the formation and characteristics of a QGP are not applicable to the real dynamical situation.

\section{Formation of a Quark Gluon Plasma}

The first question one must address is that even if present ideas on the conditions necessary for formation of a QGP are approximately correct, does the actual dynamical situation at RHIC allow them to be achieved and if so how.

We have from the early days of RHIC planning, had serious reservations in regard to this[4,5]. Although many theoretical calculations assume that central collisions of heavy ions can be described by employing local thermodynamic and chemical equilibrium which adjusts adiabatically as the collision zone develops in space and time, one can have serious reservations as to whether the collision dynamics allow and whether sufficient time exists for reaching such overall thermodynamic and chemical equilibrium.

A second approach has been to recognize that it is unlikely that thermalized, conditions can describe the whole collision dynamics in particular the phase transition itself, and thus if new phenomena (QGP) etc. occur, they form under inherently non equilibrium conditions. This scenario has been suggested and strongly emphasized by Van Hove[6,7].

Van Hove's scenario would lead to local droplets of QGP if QGP is formed. As the droplets expand they could, in some cases separate into smaller droplets. It was expected that these QGP droplets could hadronize by deflagration since this appeared to be the more likely of the two possible explosive phenomena as it was favored by entropy considerations [6-8].

It should be noted that these non-equilibrium treatments have assumed that the chemical potential is zero (i.e. baryon no. $B \approx 0$ ) and thus are directly applicable to the central region. At RHIC $100 \mathrm{Gev} / \mathrm{n} \mathrm{Au}$ on $100 \mathrm{Gev} / \mathrm{n} \mathrm{Au}$ central events are expected to have B close enough to zero for our calculational purposes, especially in the Quark-gluon plasma bubbles. It is expected that the central region at RHIC will have $B \approx 0$, however Van Hove's approach may even be qualitatively correct for the behavior of plasma droplets originally formed even in baryon dense regions, since they rely mainly on the existence of a large amount of latent heat and latent entropy in the phase transition, conditions which also apply to baryon dense regions.

If plasma droplets (possibly after breaking up into two or more droplets) hadronize by deflagration, Van Hove's scenario concluded that the resulting distribution of hadrons should show maxima approximately at the rapidities of the droplets. The expected width of the maxima was estimated to be $\sim 1$ rapidity unit and have angular distributions characteristic of a deflagration occurring in the plasma droplets. He also expected the generally expected plasma signals such as enhanced strange and multistrange particles, enhanced anti particles, enhanced lepton pair production, etc. 
If QGP is formed it may be easier to conceive of Van Hove's local droplet scenario occurring since the long times required for overall thermodynamic and chemical equilibrium to be attained may not be attainable.

If bubbles in the VAN HOVE sense are not created, the techniques described in this paper will still be applicable if localized in rapidity regions, chunks of QGP are created by any mechanism and the hadronic signals from them are not too degraded by subsequent interactions. Of course if many droplets (or chunks of rapidity localized QGP) are formed over large regions one might approximate this situation by assuming thermodynamic and chemical equilibrium over a large region. It should also be noted, as we will be discussing, that the droplets (or rapidity localized) chunks of QGP will be expected to generate very striking signals which are detectable and could provide convincing evidence for a QGP.

In this regard one should note that a bubble or rapidity localized region in QGP will be surrounded by non-QGP background, and thus allow a comparison of QGP and non-QGP backgrounds in the same event. This is a potent way to possibly establish a QGP. One should also note that these bubble like events only need to happen and their characteristics survive some of the time (even rarely) provided they provide sufficient statistics in order to provide powerful evidence for a QGP.

\section{Event Generation}

One must use an event generator suitable for estimating the non plasma events, and as a second step embed the plasma bubbles formation and deflagration, and compare plasma regions in the event with the non-plasma regions, and then also compare non plasma events with the plasma events to determine the detectability of plasma bubbles, and whether they would be striking enough to at least provide credible evidence for plasma formation, or hopefully even convincing evidence for a QGP.

\section{RHIC::EVENT - a non plasma generator}

The geometry of the A-A collision is taken into account by populating nucleons in the target and the projectile systems according to a Woods-Saxon Distribution. The nucleons have a Gaussian distribution of Fermi momentum with a small $\sigma$ of $200 \mathrm{MeV} / \mathrm{c}$. The nucleons are off the mass shell, with an average binding energy of $8 \mathrm{MeV}$. A collision interaction between a projectile, and target nucleon occurs when the distance of closest approach is less than

$\sqrt{\sigma} / \pi$ with $\sigma=33 \mathrm{mb}$. Thus this is 1.025 Fermi-the approximate size of the proton. The center of mass system of the $N-N$ collision is used.

ISAJET[9] - an event generator for high energy $N-N$ interactions, has been successful in explaining these interactions, and is therefore used as the basis of building RHIC:: EVENT. A high energy Nucleus+Nucleus (i.e. A+A) collision is represented as resulting from a series of nucleon-nucleon collisions but taking into account that after a nucleon suffers a collision 
it becomes a forward going diquark.

The MINBIAS routine of ISAJET, is based on inclusive high energy $N-N$ interactions forming multi-pomeron chains, with each chain fragmenting according to the Field-Feynman algorithm[10].

MINBIAS is used to compute the energy loss of the colliding nucleons and the produced particles . Unlike earlier event generators based on ISAJET (i.e. HIJET[11]) instead of using the leading baryon for the diquark for the next collision, RHIC::EVENT sums up the momentum of the particles produced by MINBIAS that one wants to associate with the diquark cluster and RHIC::EVENT does not allow the leading diquark to change flavors. It is speculated that the forward going cluster is what the fragmentation region is. The larger the cluster size and momentum the more RHIC::EVENT creates nuclear transparency. This algorithm can generate a very nice flat central region plateau for RHIC energies which is flat over $\approx 5$ units of rapidity.

A popular event generator, HIJING[12], is a Monte Carlo model which combines Fritiof[13] for soft beam jet fragmentation, and Pythias[14] for semi-hard mini jet Physics[15]. Gyulassy[15] shows in his Fig. 1 that HIJING reasonably represents the number of charged particles per unit $y$ or $\eta$ as a function of $y$ or $\eta$ for central-i.e. very high multiplicity events. When HIJING is applied to $100 \mathrm{GeV} /$ nucleon Au colliding with $100 \mathrm{GeV} /$ nucleon Au Gyulassy shows that for "central events" at RHIC (his Fig. 1) a flat plateau is formed centered around midrapidity or $\eta$ and extends (within $\sim 20 \%$ from the peak value) for a total of approximately four $y$ or $\eta$ units, and thus is qualitatively in agreement with the results of our RHIC::EVENT generator with leading cluster equal to only the diquark. Gyulassy points out that HIJING results are similar to those obtained with other models[13-20].

It should be noted that uncertainty originates from the poorly known early evolution of the mini-jet plasma, and this affects the height of the plateau in the calculations of Gyulassy[15]. Computing the early evolution of the color fields more reliably will allow better estimation of the plateau height.

\section{$5 \quad$ RHIC::PLASMA - a spherical plasma bubble(s) generator}

Plasma formation and decay is a very uncertain process and our first model of this phenomenon is based on work of L. Van Hove[6,7]. Van Hove's model predicted that the deconfinement transition is described in terms of formation of QCD strings in the expanding plasma. These strings in the plasma are stopped from expanding because of string tension and because of string breaking droplets or bubbles are formed. These bubbles hadronize by deflagration at the phase transition through their outer surface by ejection of low pressure hadron gas with velocities associated with the critical temperature. This process creates a rapidity distribution $d n / d y$ or a pseudorapidity distribution $d n / d \eta$ of hadrons with isolated maxima of width $\delta y$ or $\delta \eta \sim 1$ or in our case high bumpy regions $\sim 2$ units of rapidity wide on an event-by-event basis. 
The generator RHIC::PLASMA takes the regular RHIC::EVENT generation and Van Hove type[6,7] spherical plasma bubbles generated by a regional tagging scheme which takes particles from the intial RHIC::EVENT collisions and converts their energy and baryon number to a plasma bubble. The tagging region is defined as a circular cross sectional area located within the center of the overlap region with radius $R_{\text {tag }}$. Each region is then subdivided in phase space by a longitudinal momentum cuts (three bubbles can be defined in present code). The bubble has an intial energy and baryon number, where its rest frame is given by the total momentum energy four vector of the sum of the tagged particles that make up the bubble. The thermal dynamics of the bubble is generated in accord with the model of Koch, Muller and Rafelski [21]. Initially the tagged volume is used to define the chemical potential and thus the quark and gluon populations for a plasma sitting at its critical temperature. Reference [21] has worked out these equations up to a temperature of $170 \mathrm{MeV}$. We expand the volume until we get a self consistent condition that satisfies energy and baryon number between the tagged particles and the plasma bubble. This self consistent condition is equal to the number of quarks, anti-quarks and gluons $\left(N_{q} N_{\bar{q}} N_{g}\right)$. $N_{s}$ and $N_{\bar{s}}$ are the strange quark and anti-quark numbers where $N_{q}$ and $N_{\bar{q}}$ are the light quarks $(u, d)$. When hadronization takes place gluons fragment into quarks and anti-quarks leading to an effective number given by $\tilde{N}_{q}=N_{q}+f_{q} N_{g} ; \tilde{N}_{\bar{q}}=N_{\bar{q}}+f_{q} N_{g} ; \tilde{N}_{s}=N_{s}+f_{s} N_{g} ; \tilde{N}_{\bar{s}}=N_{\bar{s}}+f_{s} N_{g}$, where $f_{q}=$ gluon fragmentation function for $u$ and $d$ quarks and $f_{s}=$ gluon fragmentation function for $s$ quarks. The average number of particles produced each with a Boltzman distribution corresponding to the critical temperature is: $N_{\pi}=\alpha \tilde{N}_{q} \tilde{N}_{\bar{q}}, N_{K}=\alpha \tilde{N}_{q} \tilde{N}_{\bar{s}}$, $N_{\phi}=\alpha \tilde{N}_{s} \tilde{N}_{\bar{s}}, N_{n}=\frac{1}{3 !} \beta \tilde{N}_{q} \tilde{N}_{q} \tilde{N}_{q}, N_{\bar{n}}=\frac{1}{3 !} \beta \tilde{N}_{\bar{q}} \tilde{N}_{\bar{q}} \tilde{N}_{\bar{q}}, N_{\Lambda}=\frac{1}{2 !} \beta \tilde{N}_{q} \tilde{N}_{q} \tilde{N}_{s}, N_{\bar{\Lambda}}=\frac{1}{2 !} \beta \tilde{N}_{\bar{q}} \tilde{N}_{\bar{q}} \tilde{N}_{\bar{s}}$, $N_{\Xi}=\frac{1}{2 !} \beta \tilde{N}_{q} \tilde{N}_{s} \tilde{N}_{s}, N_{\bar{\Xi}}=\frac{1}{2 !} \beta \tilde{N}_{\bar{q}} \tilde{N}_{\bar{s}} \tilde{N}_{\bar{s}}, N_{\Omega}=\frac{1}{3 !} \beta \tilde{N}_{s} \tilde{N}_{s} \tilde{N}_{s}, N_{\bar{\Omega}}=\frac{1}{3 !} \beta \tilde{N}_{\bar{s}} \tilde{N}_{\bar{s}} \tilde{N}_{\bar{s}}$

$\alpha$ and $\beta$ are given by the equations:

$$
\begin{aligned}
& \alpha=\frac{4 Q_{1}}{\left(3 Q_{1}^{2}+Q_{2}^{2}\right)} \\
& \beta=\frac{8}{\left(3 Q_{1}^{2}+Q_{2}^{2}\right)}
\end{aligned}
$$

where $Q_{1}=\tilde{N}_{q}+\tilde{N}_{\bar{q}}+\tilde{N}_{s}+\tilde{N}_{\bar{s}}$ and $Q_{2}=\tilde{N}_{q}-\tilde{N}_{\bar{q}}$. These equations make sure baryon number is conserved and particles are formed randomly out of the particle densities. The above particles then are generated with a Boltzman distribution of a $170 \mathrm{Mev}$ temperature spherically in phase space. The spherical distribution creates a bump in rapidity or pseudorapidity of the type that Van Hove[6,7] predicted. However, the width of the bumps is about 2 units. The geometry of the expansion of the plasma bubble or fireball is dependent on QCD and the collision dynamics. Other cases will be considered subsequently.

\section{RHIC::LANDAU}

Although we have so far considered spherical bubbles, any reasonable bubble shape can be incorporated in our plasma generator. There is a one-to-one correspondence between the bubble shape assumed and the effect on the rapidity distribution and structure in it caused 
by the bubble. Given a particular observed rapidity distribution, assumed to be due to plasma bubble(s) formation, we can also deduce general characteristics of the shape of the plasma regions. For example the shapes of three other localized in rapidity QGP regions (or bubble shapes), are treated below.

The decision as to whether to attribute an experimentally observed bubble-like phenomenon to plasma formation will depend on the behavior and the correlation of the various possible plasma signals associated with it (some of which are shown in the figures), other characteristics of the data, all at the time known facts, and very importantly the lack of a viable other conventional physics alternative.

In particle and nuclear physics the fireballs (not in any way shown to contain QGP) that have been observed, have an additional one dimensional or longitudinal expansion left over from their production mechanism (Landau fireball[22]). The program RHIC::LANDAU takes the regular RHIC::PLASMA program and replaces the spherical expansion of a QGP bubble by an expansion that is consistent with thermal fits done on SS collisions at 200 $\mathrm{GeV} / \mathrm{c}$ per nucleon incident on a target[23]. The width of the rapidity peak distribution changes from a two unit spread to a four unit spread, where $50 \%$ of the tagged energy goes into particle production and $50 \%$ goes into longitudinal expansion. In the language of Van Hove the string stopping due to breaking is not complete, so that longitudinal expansion is left in the individual strings or bubbles.

\section{RHIC::SMOKE}

The plasma bubbles that we have generated give structure to the rapidity or pseudo-rapidity spectrum of the produced particles. This structure is directly related to the geometry of the expansion. The geometry which has been observed in experiments is the elliptical expansion of the Landau fireball, however, sharp structure in pseudo-rapidity has been observed in cosmic ray experiments[24]. We can make our plasma bubbles produce sharp structures by changing an elliptical or watermelon shape to a flat or pancake shape. We do this only as a phenomenological manipulation of our code. The end process is a ring of particles emitted at a fixed angle to the beam axis much like an expanding smoke ring. Since these particles have nearly a fixed angle they lead to sharp pseudo-rapidity bumps of the intermittent type seen in cosmic ray data[24].

\section{RHIC::CHIRAL}

Another novel event structure seen in cosmic ray data[25]is the so-called "centauro-like" and "anti-centauro-like" fluctuations of charged and neutral particles. A proposed explanation of these events relies on a chiral phase transition. Rajagopal and Wilczek[26] proposed a "quench" scenario in which the hadronic condensate after a phase transition is initially chirally symmetric, but its evolution is taken to follow classical equations of motion at zero temperature. The non-equilibrium dynamics of the chiral transition (using a linear $\sigma$ model 
to describe the collective chiral behavior) in relativistic heavy ion collisions yields large disoriented chiral condensates. These disoriented chiral condensates lead to the non-Poisson distribution of the "centauro-like" and the "anti-centauro-like" events. The probability distribution of neutral pions $P(R 3)[27]$, where $R_{3}=\frac{N_{\pi^{0}}}{\left(N_{\pi^{+}}+N_{\pi^{0}}+N \pi^{-}\right)}$, is equal to $P\left(R_{3}\right)=\frac{1}{\sqrt{R_{3}}}$. In contrast, typical hadronic collisions produce a binomial distribution of $R 3$ peaked at the isospin symmetric value of $1 / 3$.

RHIC::CHIRAL generates the above like structures using the RHIC::PLASMA bubble code but making the bubble hadronize into a $70 \mathrm{MeV}$ temperature pion gas with a $R_{3}$ distribution chosen to be that of the disoriented chiral condensate model. In a given $\mathrm{Au}-\mathrm{Au}$ event each bubble has an independent $R_{3}$.

It is obvious that there is a relationship between structures in the rapidity distribution and the geometry of the plasma bubbles, which cause them. Thus if a certain type of structure is observed in the future experiments, this will infer the geometry characteristics of the bubble region (or whatever) caused it.

\section{Event Generation and Detection}

We believe the STAR Detector[28], for example, is suitable for investigating our predictions. Any statements about STAR are our own estimates (also based on estimates of others) merely used for comparison to our prediction purposes, and should, therefore, be taken as an approximation.

The high multiplicity expected in STAR central (and some non central) events coupled with the almost complete solid angle coverage over a substantial central rapidity range for $100 \mathrm{GeV}$ per nucleon gold on gold down to $\mathrm{p}+\mathrm{p}$ collisions opens up the possibility of observing single events well enough to decide whether equilibrium, non-equilibrium or strikingly different events occur individually. This eliminates the possibility of false conclusions being drawn in inclusive studies which may average over events in such a fashion as to eliminate their most striking and important characteristics. This could be a crucial element in establishing that a Quark-Gluon Plasma is produced at RHIC or observing other new phenomena which may even violate QCD.

The availability of as full solid angle particle identification as practical over a substantial particle momentum range will be of considerable aid in this program.

We believe the study of single events on an event by event basis is a most potent approach to search for a quark gluon plasma or whatever else nature reveals at RHIC ie perhaps new physics beyond QCD.

Some results of our calculations for $100 \mathrm{GeV} /$ nucleon AU colliding with 100 $\mathrm{GeV} /$ nucleon Au single event central collisions at RHIC:

The STAR detector is the most appropriate for our approach, hence the following calculations were made for it using the geometric acceptance of the central TPC detector, and 
particle identification, by $d e / d x$ (ionization loss), and the planned Time-of-Flight (TOF) covering the central TPC. Efficiencies for tracking and particle identification were not included.

Figures 1-4 show the calculated results for a single RHIC::Event. The plasma induced rapidity bumps you see in the Figs.(5-8) are based on choosing $R_{t a g} \approx 1.3 \mathrm{fm}$ which leads to the very modest assumption that only $4.5 \%$ of the available energy in a central collision is converted to QGP (as described in the event generator section RHIC::PLASMA).

Please note that if $R_{t a g}$ were chosen to be $\approx 2.5 \mathrm{fm}$, which would lead to $\approx 15 \%$ of the available energy being converted to QGP, the rapidity bump amplitudes over background would increase by about a factor of three and the statistics (i.e. number of particles in the rapidity bump) would increase by about a factor of three (i.e. the bump height relative to the background and the number of particles in the bump would scale up approximately as the percentage of energy converted to QGP [that is increase by the factor (\% energy of QGP)/4.5\%.] The figures for $R_{t a g} \approx 2.5 \mathrm{fm}$ (figs. 9-13) which converts $\approx 15 \%$ of the available energy to QGP show truly dramatic $d n / d \eta$ vs. $\eta$ peaks for single "central" events.

\section{RHIC::PLASMA - spherical bubbles}

(a) The Plasma bubble is produced at central rapidity by $100 \mathrm{GeV} / \mathrm{n}$ Au colliding with $100 \mathrm{GeV} / \mathrm{n} \mathrm{Au}$ (results figs. 5-8) in the region with $R_{t a g} \approx 1.3 \mathrm{fm}$ which contains only approximately $4.5 \%$ of the available energy. Even this conservative estimate will as, we can see from (Figs. 5-8), produce striking signals in these single events.

(b) The plasma bubble is produced at central rapidity where the $R_{t a g} \approx 2.5 \mathrm{fm}$ which contains $\approx 15 \%$ of the available energy. These assumptions lead to truly dramatic signals for single events (see figs. 9-13).

(c) Figures (14-16 show the psuedorapicity distribution of dET/dNch (average energy per charged particles) for the three cases considered. The increase in ET for the plasma bubbles is attributable to the fact that the plasma contains much larger percentages of heavy hadrons such as protons, anti protons, and charged kaons, that the cascade (non-plasma events), and the plasma hadronizes at $170 \mathrm{Mev}$. These results come right out of the model with no extra assumptions. We can generate an enormous number of detailed plots of desired quantities, but this is obviously not suitable for a publication, so that we must be selective. However when data is obtained with rhic we will generate what is needed and relevant to confront our model, and in fact keep an entirely open mind on the subject, and modify our aproach to the extent necessary to understand the data, and others may well do the same.

No one can predict what RHIC will reveal with any credible assurance. This paper takes a different approach and therefore will be of vlaue whether it fits the data or not.

It should be noted that if multiple bubbles are created in a single event, each will result in rapidity bumps, strangeness, and anti-baryon-baryon enhancements etc. in that rapidity interval, which corresponds to that bubble. Localized QGP bubble formation, probably 
depends on a first order transition, however it should also be expected that any unusual occurrence in a local rapidity region which survives to the end state will show up approximately in that rapidity region in the final single event. Of course if QGP is made abundantly over large regions of an event our bubble techniques will become insensitive. But it is considered unlikely this will occur in all events, and we can study those events where plasma regions (or bubbles are localized).

Of course, single events using various selection criteria, can be lumped together for statistical and overall view and analysis reasons. However in doing this great care must be taken to avoid introducing unwanted biases in the result.

Finally, these results are to be taken only as an indication of the promise of the possibility of striking signals of a QGP occurring in single events and being detected in STAR. The correlations of the pions, kaons, protons, and anti-protons, and the detailed characteristics of the events will of course be important. Our approach and ideas will of course change as detailed data is obtained and analyzed. We will keep an open mind as the observations come in.

It is important to note that various energies and various beam nuclei from $\mathrm{Au}+\mathrm{Au}$ down to $\mathrm{p}+\mathrm{p}$ will be used and these results will impact on any final conclusion. Furthermore it is not necessary to explain every observation. Finding striking and unusual events some of the time could well lead to establishment of a QGP if it naturally explains them, and no viable alternate explanation is found, and there is no contradiction of the QGP evidence by other characteristics of the data.

The event generator VNI[29] will also be used in future work. VNI is Monte-Carlo eventgenerator for leptons, hadron, and nucleus collisions on each other. It uses the real-time evolution of parton cascades in conjunction with a self-consistent hadronization scheme, as well as the development of hadron cascades after hadronization. The parton cascading in nucleus-nucleus collisions leads to space-time regions of high energy density. These regions can become the source of plasma bubbles like the ones described above. In the future we plan to use VNI for further work on bubbles. We plan to use VNI as a source of bubble production. However we must address the problem of how to determine or estimate the energy transmitted to the bubble by non-perturbative QCD interactions. VNI will place the bubble in a hadronic final state which could give a realistic transport of its emitted hadrons to the final detection of plasma signals.

New unexpected phenomena beyond QCD could be observed if they occur at RHIC, and in that event we plan to use our own and newly developed event generators to investigate them. It is interesting to note that a recently proposed bubble phenomenon[30,31] as described below can be investigated with our techniques and generators. Any assumptions about STAR performance, such as DE/DX, planned time-of-flight performance, etc. are to be considered only as our personal estimates for the purpose of comparison with our event generator predictions. 


\section{Odd CP Bubbles of Metastable Vacuum}

Kharzeev and Pisarski[30] expect that bubbles of metastable vacua with odd CP will induce a net flow of pion charge. This flow can be modeled with a parallel chromo electric and magnetic field. A quark traveling in this field will drift up in a spiral, while an anti-quark will drift down. These added impulses will end up in positive charged pions traveling up and negative charged pions traveling down. Kharzeev[31] has estimated an average impulse of $30 \mathrm{MeV} / \mathrm{c}$ for a quark crossing the diameter of the bubble.

If we place the electric $\vec{E}$ and the magnetic $\vec{B}$ field along the $x$-axis, then we can write down the added impulse for positive pions $\left(\pi^{+}\right)$as vector equations 1 :

$$
\begin{gathered}
P_{p x}^{\prime}=P_{p x}+P_{E} \\
P_{p y}^{\prime}=P_{p y}-\frac{P_{B} P_{p z}}{P_{p T O T}} \\
P_{p z}^{\prime}=P_{p z}+\frac{P_{B} P_{p y}}{P_{p T O T}},
\end{gathered}
$$

where $\overrightarrow{P_{p}^{\prime}}$ is the charged $\pi^{+}$momentum $\vec{P}_{p}$ is the $\pi^{+}$momentum without the added effect and $P_{p T O T}$ is the magnitude of the momentum $\vec{P}_{p}$. $P_{E}$ is the impulse which comes from the electric field acting, while $P_{B}$ comes from the magnetic field. Vector equations 2 for negative pions $\left(\pi^{-}\right)$is:

$$
\begin{gathered}
P_{m x}^{\prime}=P_{m x}-P_{E} \\
P_{m y}^{\prime}=P_{m y}+\frac{P_{B} P_{m z}}{P_{m T O T}} \\
P_{m z}^{\prime}=P_{m z}-\frac{P_{B} P_{m y}}{P_{m T O T}},
\end{gathered}
$$

where $p$ for positive has been replaced by $m$ for minus. Also the sign of the impulse has changed.

We will model the added impulse by assuming that we start with a bubble of radius $r_{0}$ that has quarks uniformly distributed inside the bubble.

The point $P$ of the quark inside the bubble is given by equation (3):

$$
\begin{gathered}
x_{p}=r_{p} \sin \theta_{p} \cos \phi_{p} \\
y_{p}=r_{p} \sin \theta_{p} \sin \phi_{p} \\
z_{p}=r_{p} \cos \theta_{p},
\end{gathered}
$$

where $x_{p}, y_{p}$, and $z_{p}$ are the Cartesian coordinates and $r_{p}, \theta_{p}$, and $\phi_{p}$ are the spherical coordinates. From this point $P$ the quark travels in a line given by its momentum

$$
x=\frac{P_{x}}{P_{z}}\left(z-z_{p}\right)+x_{p}
$$




$$
y=\frac{P_{y}}{P_{z}}\left(z-z_{p}\right)+y_{p}
$$

where $P_{x}, P_{y}$, and $P_{z}$ are the momentum components of the quark. The quark will pass through the surface of the bubble when

$$
r_{0}^{2}=x^{2}+y^{2}+z^{2}
$$

We now substitute 4 into 5 , we have

$$
r_{0}^{2}=\left(\frac{P_{x}}{P_{z}}\left(z-z_{p}\right)+x_{p}\right)^{2}+\left(\frac{P_{y}}{P_{z}}\left(z-z_{p}\right)+y_{p}\right)^{2}+z^{2}
$$

Equation (6) can be rewritten in quadratic form using $z^{\prime}$

$$
\left(\frac{P_{x}^{2}}{P_{z}^{2}}+\frac{P_{y}^{2}}{P_{z}^{2}}+1\right)\left(z^{\prime}\right)^{2}+2\left(\frac{P_{x} x_{p}}{P_{z}}+\frac{P_{y} y_{p}}{P_{z}}+z_{p}\right)\left(z^{\prime}\right)+r_{p}^{2}-r_{o}^{2}=0
$$

where $z^{\prime}=z-z_{p}$.

Using vector notation equation (7) becomes

$$
\frac{P_{p}^{2}}{P_{z}^{2}}\left(z^{\prime}\right)^{2}+\frac{2}{P_{z}}\left(\vec{P}_{p} \cdot \vec{r}_{p}\right)\left(z^{\prime}\right)-\left(r_{o}^{2}-r_{p}^{2}\right)=0
$$

Thus we have two solutions:

$$
z^{\prime}=z-z_{p}=\frac{\left(\vec{P}_{p} \cdot \vec{r}_{p}\right) \pm \sqrt{\left(\vec{P}_{p} \cdot \vec{r}_{p}\right)^{2}+P_{p}^{2}\left(r_{o}^{2}-r_{p}^{2}\right)}}{P_{p}^{2}}
$$

because a line strikes a sphere twice when passing through it.

The distance the quark travels is given by

$$
\text { dist }=\sqrt{\left(x-x_{p}\right)^{2}+\left(y-y_{p}\right)^{2}+\left(z-z_{p}\right)^{2}} .
$$

Using equation (4), the distance equation (10) becomes:

$$
\text { dist }=\sqrt{\frac{P_{x}^{2}}{P_{z}^{2}}+\frac{P_{y}^{2}}{P_{z}^{2}}+\frac{P_{z}^{2}}{P_{z}^{2}}}\left|\left(z-z_{p}\right)\right|
$$

or

$$
\text { dist }=\frac{P_{p}}{\left|P_{z}\right|}\left|\left(z-z_{p}\right)\right|
$$

Plugging in (9) into (11), we obtain:

$$
\text { dist }=\left|\frac{\left(\vec{P}_{p} \cdot \vec{r}_{p}\right) \pm \sqrt{\vec{P}_{p} \cdot \vec{r}_{p}+P_{p}^{2}\left(r_{o}^{2}-r_{p}^{2}\right)}}{P_{p}}\right|
$$


Spherical coordinate system (3) makes equation (12) look very simple

$$
\begin{gathered}
\text { dist }=r_{p}\left(\sin \theta \sin \theta_{p}\left(\cos \phi \cos \phi_{p}+\sin \phi \sin \phi_{p}\right)+\cos \theta \cos \theta_{p}\right) \\
\pm \sqrt{r_{p}^{2}\left(\sin \theta \sin \theta_{p}\left(\cos \phi \cos \phi_{p}+\sin \phi \sin \phi_{p}\right)+\cos \theta \cos \theta_{p}\right)^{2}+r_{0}^{2}-r_{p}^{2}}
\end{gathered}
$$

where $\theta$ and $\phi$ are the angles of the momentum vector $\vec{P}_{p}, \theta_{p}$ and $\phi_{p}$ are the angles of the point $P$ with $r_{p}$ being the radius of the point and $r_{0}$ the radius of the bubble.

We now want to obtain the distribution of dist taking both solutions for every point $P$, when the points are uniformly placed inside the bubble. The easiest way to do this calculation is using Monte Carlo methods. The result of such a numerical method leads to

$$
\frac{d N}{d_{d i s t}} \propto \cos \left(\frac{\pi d i s t}{4 r_{o}}\right)
$$

The impulse that the quark gets is directly proportional to the distance. Since $2 r_{o}$ is the maximum distance, we can set the maximum impulse $\left(\Delta P_{\max }\right)$ equal to this value and easily write down the impulse distribution.

$$
\frac{d N}{d \Delta P} \propto \cos \left(\frac{\pi \Delta P}{2 \Delta P_{\max }}\right)
$$

In equations (1) and (2) the value of $P_{E}$ and $P_{B}$ can be chosen by picking a random number $R$ between zero and one and calculating

$$
\Delta P=\Delta P_{\max }=\frac{2 \Delta P_{\max }}{\pi} \cos ^{-1}(R)
$$

The next aspect in bubble simulation is the kinematics of the pions produced by the bubble. Gyulassy[32] suggested jet fragmentation would show these added impulse to their pions. Studies of changes of jet fragmentation in heavy ion events is already underway[33]. For this simulation we consider bubble kinematics that would arise from a chiral low temperature bubble or a bubble that produced pions which look very much like the regular pions of a heavy ion event. A set of programs using the event generator RHIC::PLASMA is used to simulate bubbles that have imparted impulses given by equation (16).

Chiral bubbles are spherically symmetric distributions of pions having a Boltzman energy spectrum of $70 \mathrm{meV}$ temperature. The chromo $E$ and $B$ field is randomly assumed in any direction. The E field gives a impulse to $\pi^{+}$along its direction, while the $\pi^{-}$impulse is opposite to its direction. The impulse of the $\mathrm{B}$ field is at right angles to the direction, where the example of $\mathrm{B}$ and $\mathrm{E}$ along the $\mathrm{X}$-axis is given by equations (1) and (2).

Besides a low temperature chiral bubble, let us consider a higher temperature bubble which expands in the longitudinal beam direction. The net result leads to a bubble which blends into the regular background of RHIC::EVENT. 
The program generates a bubble by a regional tagging scheme which takes particles from the initial RHIC::EVENT collision and converts the energy into a bubble. The tagging region is defined as a circular cross sectional area located within the center of the overlap region with radius $R_{t a g}$. The bubble has an initial energy, where its rest frame is given by the total momentum energy four vector of the sum of the tagged particles that are used to create the bubble.

\section{Summary and Conclusions}

In this paper we have discussed a possible way striking signals for a QGP could be generated at RHIC and detected. We have extended and generalized the original scenario proposed by Van Hove[6,7], by developing a detailed model with associated event generators to explore this scenario. Some of the results have been presented here.

If QGP bubbles or localized rapidity regions of QGP are created in some of the RHIC collisions (probably most likely for highest energy $\mathrm{Au}$ on $\mathrm{Au}$ ), and the characteristics of their hadron emissions preserved, at least substantially in part of the events final state, we have shown that reasonable event generators that we developed can provide striking signals in individual events, even under the modest assumption that only $4.5 \%$ of the available energy (corresponding to the available energy for plasma formation generated in a central collision contained within a radius of about $1.3 \mathrm{fm}$ ) forms the QGP bubble. If larger amounts of the available energy form a QGP bubble (or a localized rapidity region) the signals will scale with the QGP energy, and could become truly dramatic (see Figs. 9-13).

We considered how the STAR detector, for example, might observe some of these modest $4.5 \%$ of the available energy QGP signals (figs. 5-8). If we assume $\approx 15 \%$ of the available energy is converted to plasma, we obtain truly dramatic signals (see figs. 9-13) and indicate how the STAR detector might observe some of these.

No one can predict what RHIC will reveal with any credible assurances. However when data is obtained with RHIC we will generate what is needed and relevant to confront our model, and in fact keep an entirely open mind on the subject, and modify our approach to the extent necessary to understand the data, and others may well do the same.

This paper and the event generators we have developed take a different approach, and therefore will be of value whether it can explain characteristics of the data or not.

Finally it should be noted that our RHIC::EVENT and RHIC::PLASMA generators can be used to investigate "odd CP bubbles" and likely can be adapted to many new investigations. We also discussed how the VNI event generator could be applied to QGP formation. 


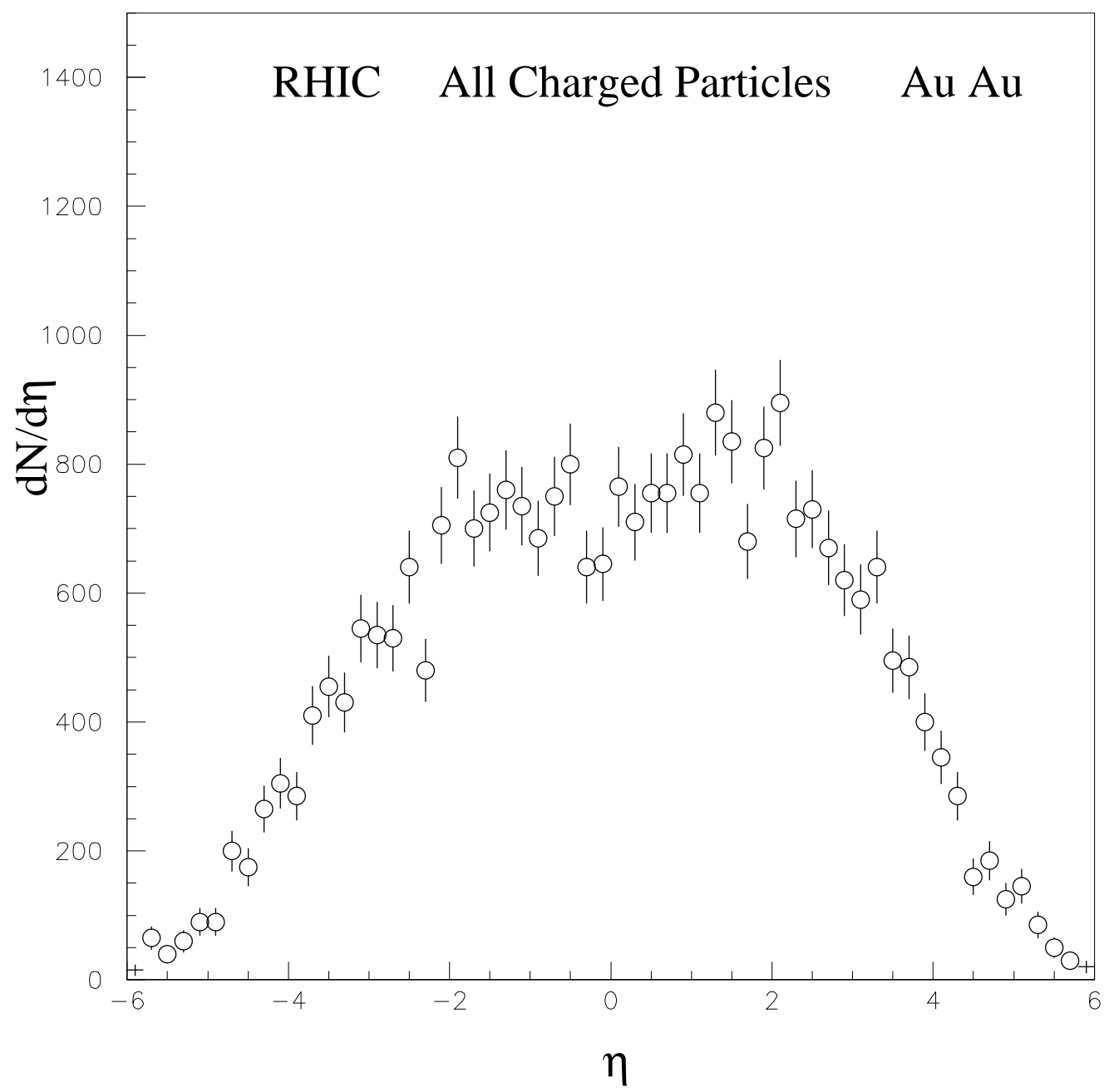

Figure 1: The generated pseudorapidity distribution of all charged particles from a single "central" $100 \mathrm{Gev} / \mathrm{n}$ AU+AU RHIC::EVENT The expected central plateau without any evidence of structure is observed. 


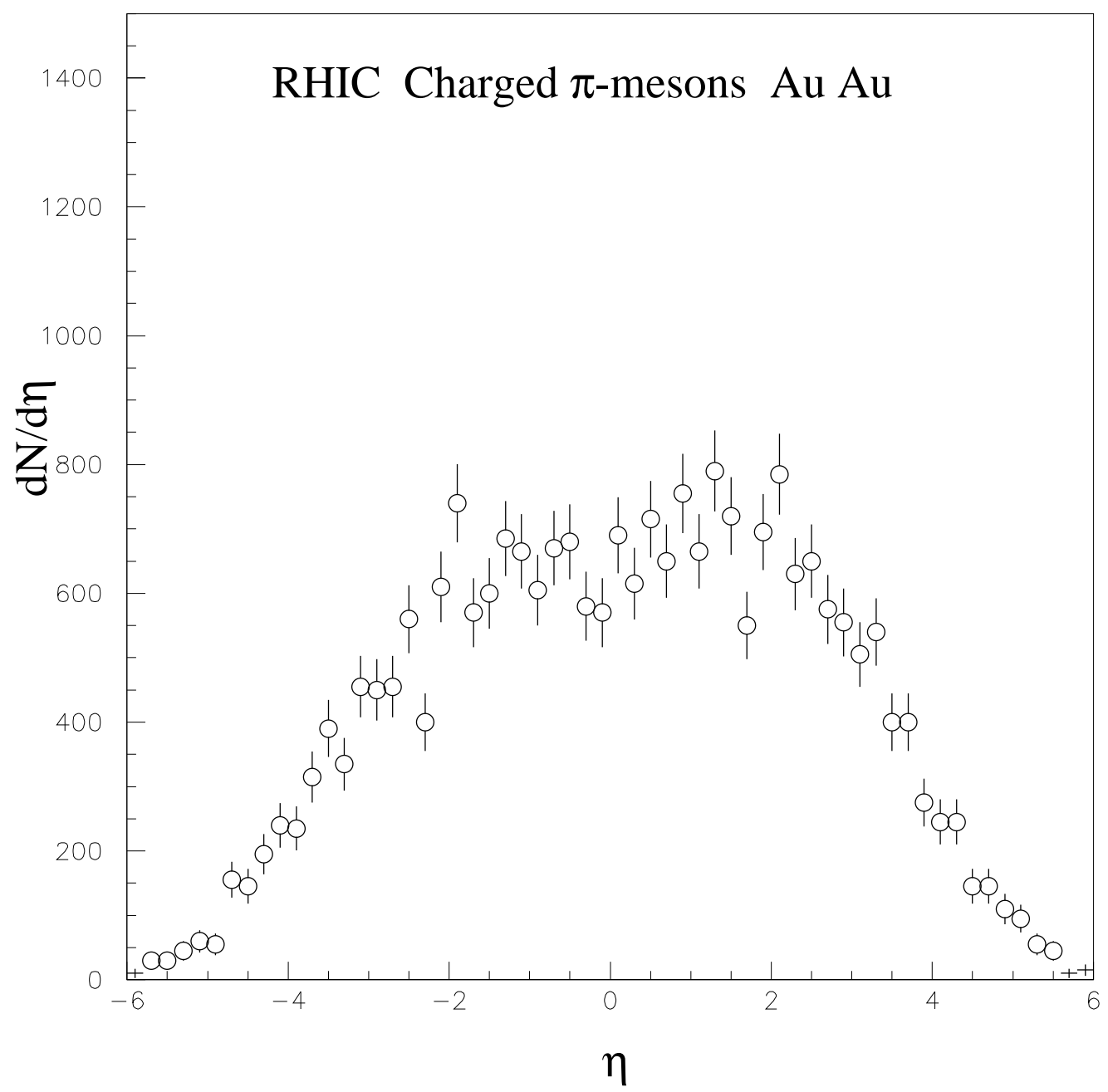

Figure 2: The generated pseudorapidity distribution of all charged pions from a single "central" $100 \mathrm{Gev} / \mathrm{n}$ AU+AU RHIC::EVENT. The expected central plateau with no evidence of structure is observed. Negative pions can be identified approximately by assuming all negative particles are pions. If one removes kaons and protons (or anti-protons) from a momentum region, what remains is almost entirely pions. 


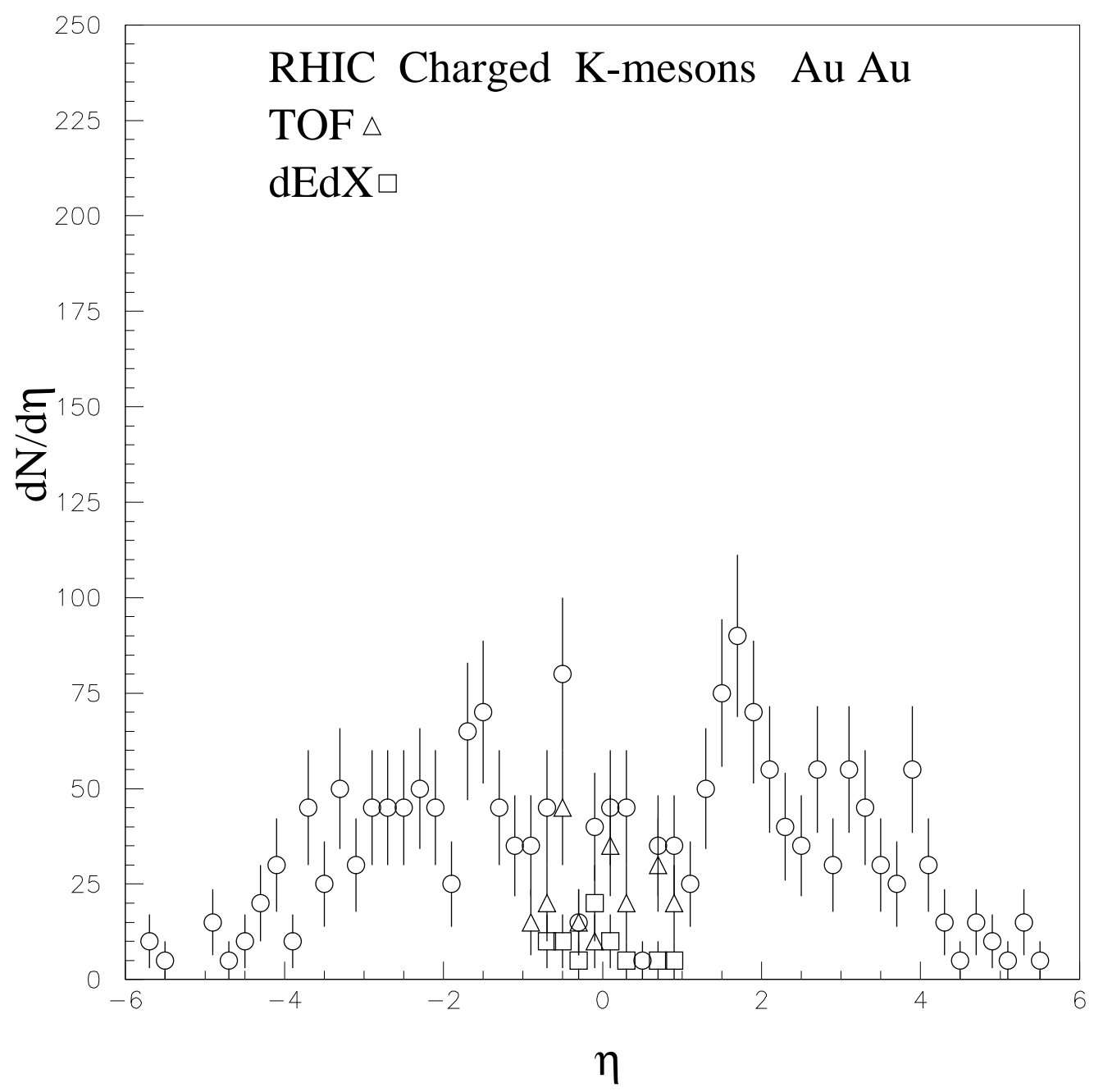

Figure 3: The generated pseudorapidity distribution for charged K-mesons from a single "central" AU+AU RHIC::EVENT. The STAR identified K-mesons estimated by DE/DX (ionization) and TOF (Time-of-Flight) with the geometric efficiency only for the STAR detector central TPC. A central plateau with no evidence for structure is observed. 


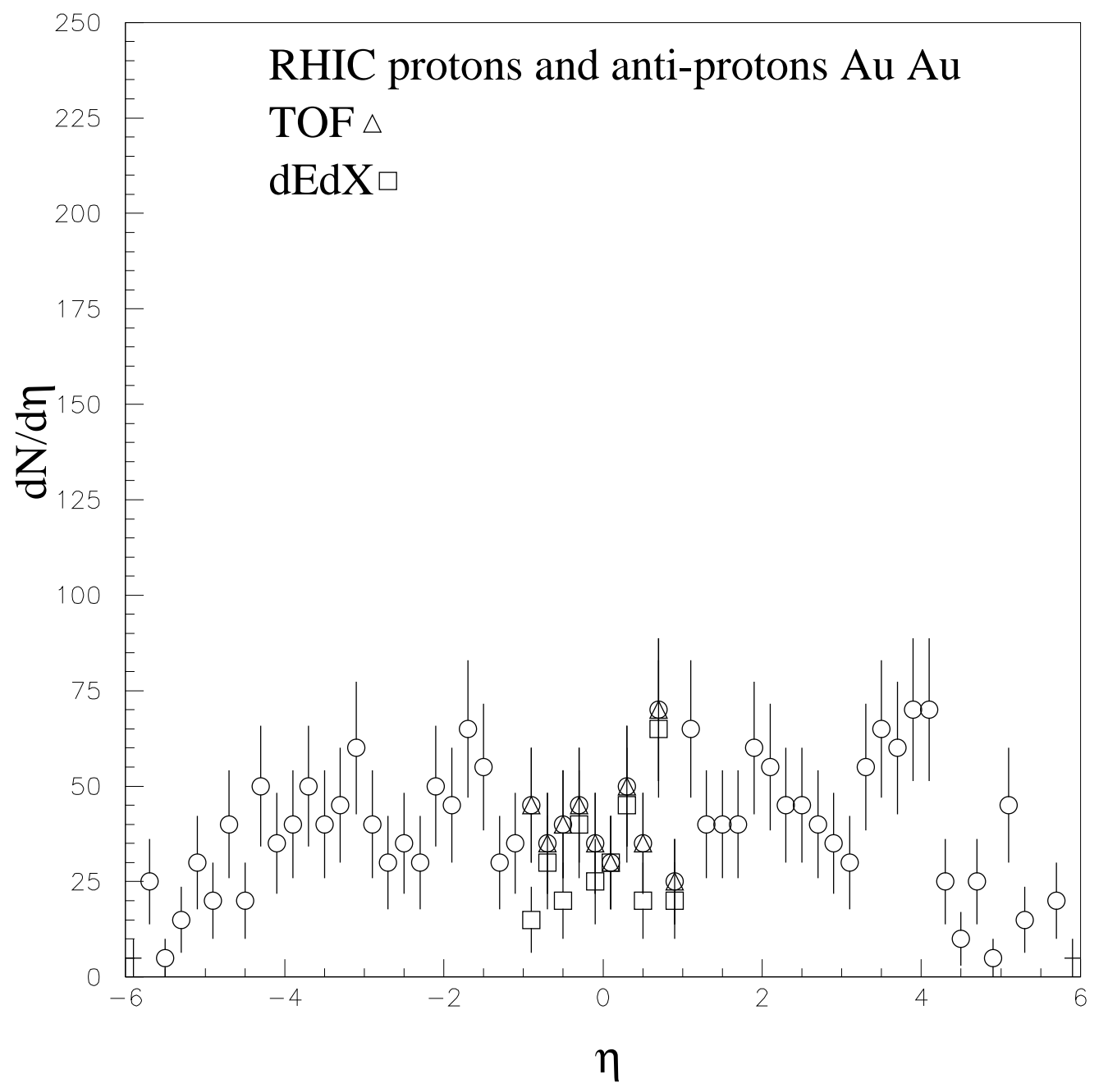

Figure 4: The generated pseudorapidity distribution for protons and antiprotons from a single "central" AU+AU RHIC::EVENT with STAR DE/DX (ionization) and TOF (Timeof-Flight) identification with the geometric efficiency only for the central TPC shown. A central plateau with no evidence of structure is observed. 


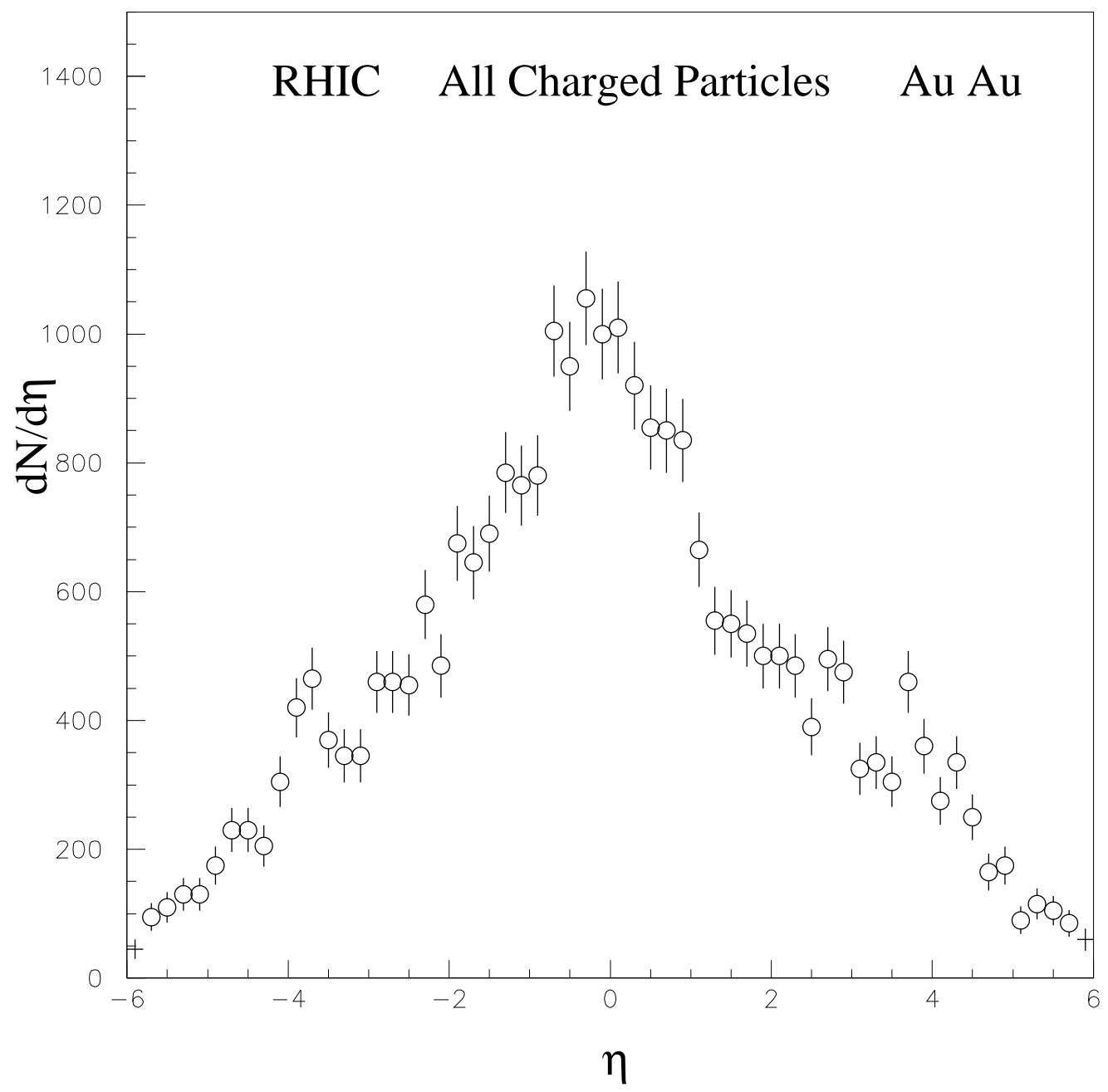

Figure 5: The generated pseudorapidity distribution of all charged particles from a single RHIC::PLASMA "central" EVENT. Note the well-defined $\eta$ central peak in contrast to the structureless result in Fig. 1 for RHIC::EVENT. The bubble energy was $4.5 \%$ of the available energy. 


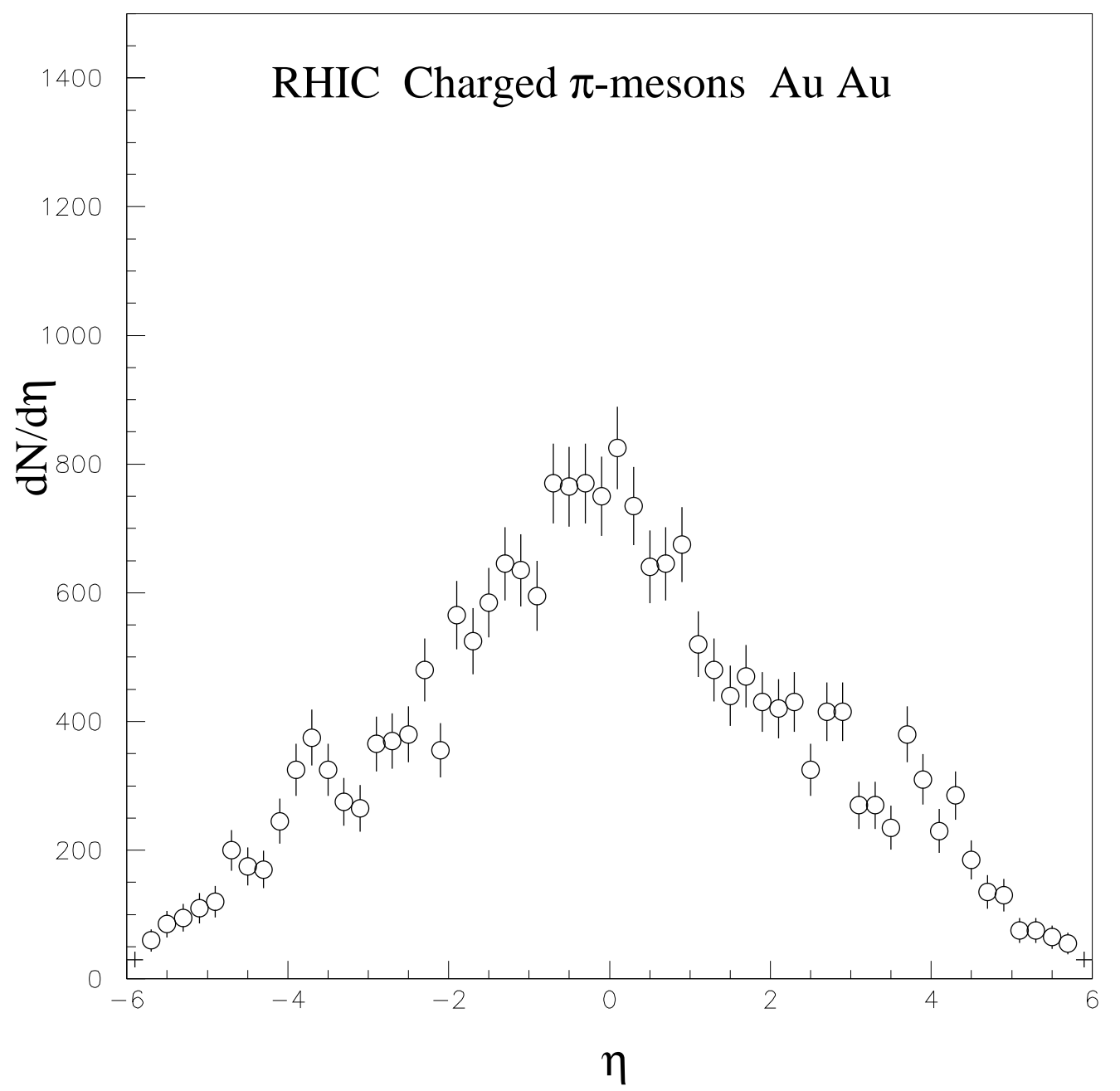

Figure 6: The generated pseudorapidity distribution for all charged pions from a single "central" $100 \mathrm{Gev/n}$ AU+AU RHIC::EVENT again showing a well defined central bubble peak. Negative pions can be identified approximately by assuming all negative paticles are pions. If one removes kaons and protons (or anti-protons from a momentum region what remains is almost entirely pions. The bubble energy was $4.5 \%$ of the available energy. 


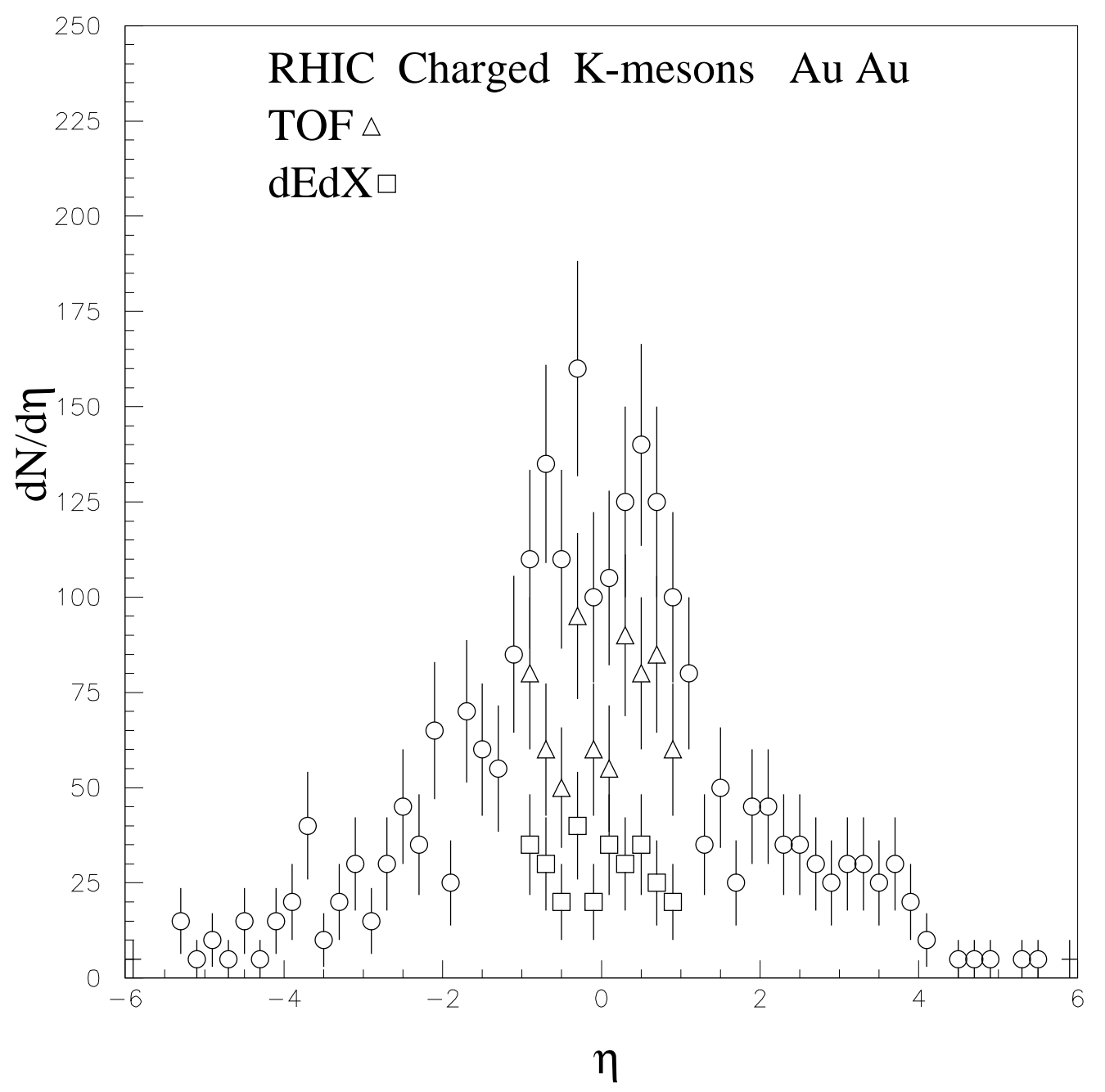

Figure 7: The generated pseudorapidity distribution for all charged K-mesons from a single "central" $100 \mathrm{Gev/n} \mathrm{AU+AU} \mathrm{RHIC::EVENT} \mathrm{showing} \mathrm{a} \mathrm{peak} \mathrm{and} \mathrm{that} \mathrm{TOF} \mathrm{can} \mathrm{be} \mathrm{effective}$ especially if larger than $4.5 \%$ of energy is converted to plasma in some events. The geometric efficiency only for the central TPC was used. The bubble energy was $4.5 \%$ of the available energy. 


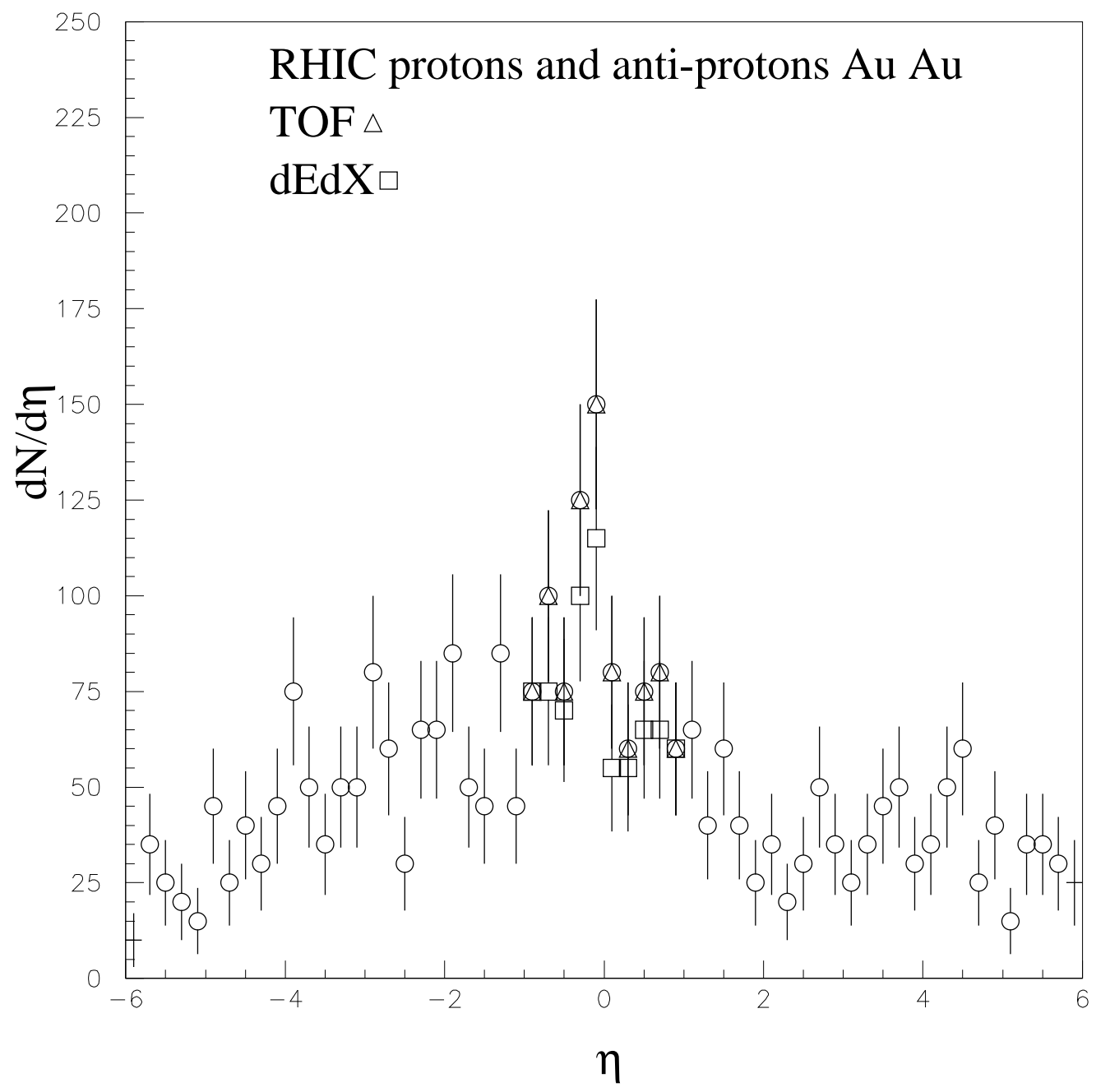

Figure 8: The generated pseudorapidity distribution for protons and anti-protons, the geometric efficiency only for the central TPC was used showing a peak which is well identified by TOF and to some extent indicated by DE/DX. The bubble energy was $4.5 \%$ of the available energy. 


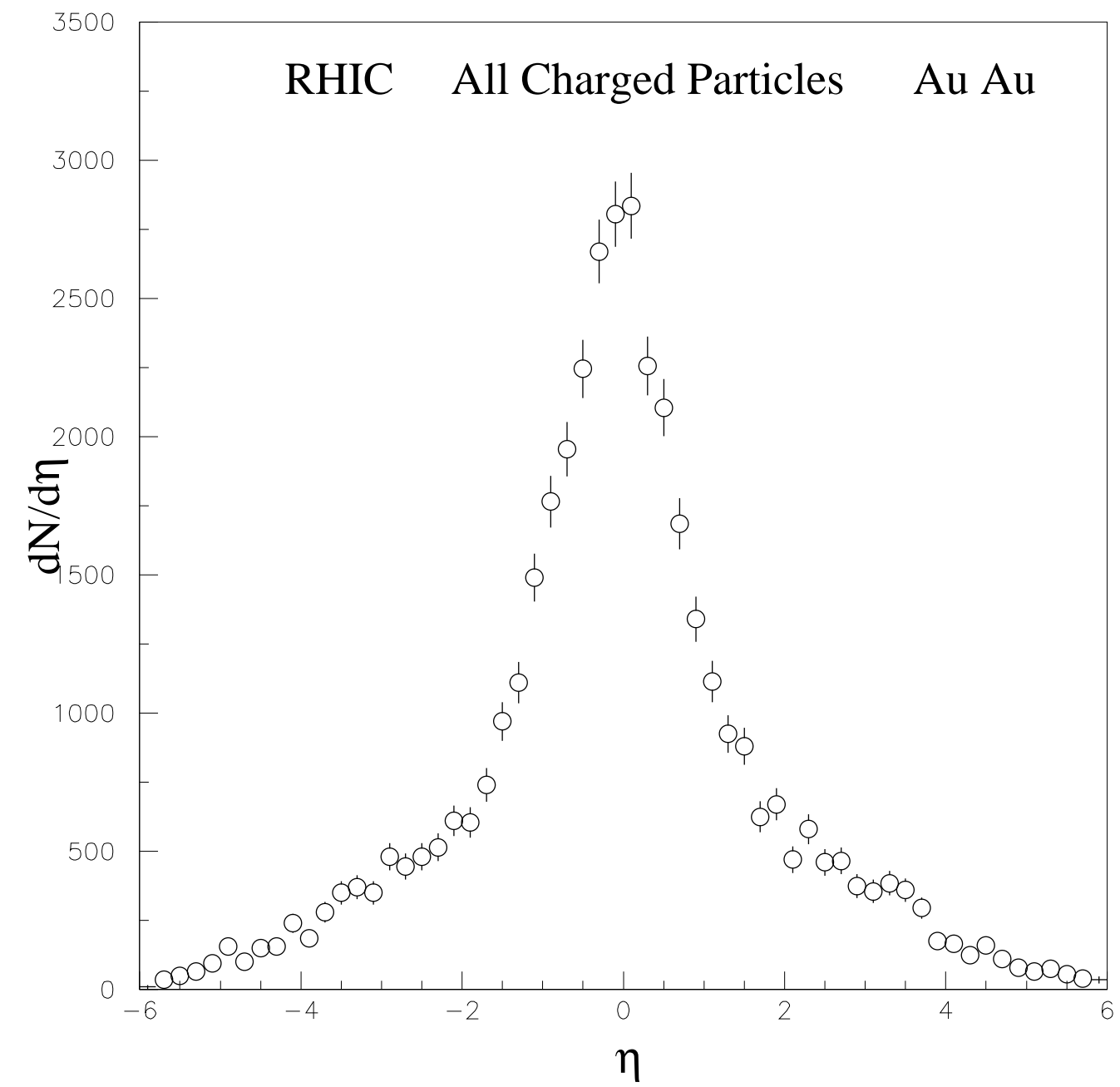

Figure 9: The generated pseudorapidity distribution of all charged particles from a single "central" $100 \mathrm{Gev} / \mathrm{n}$ AU+AU RHIC::PLASMA event. The height of the bubble peak is about four times the background level. The bubble energy was $\approx 15 \%$ of the available energy. 


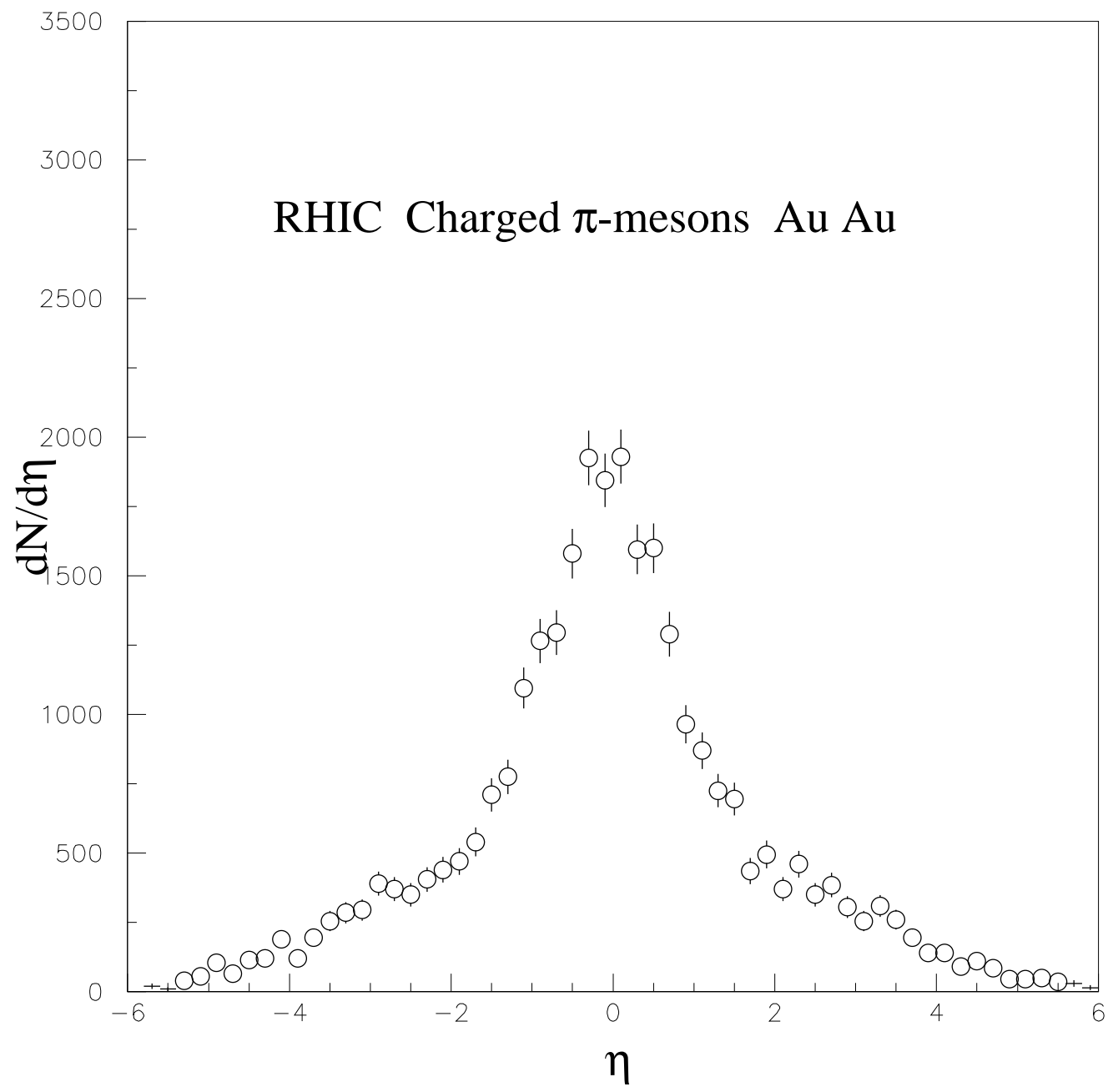

Figure 10: The generated pseudorapidity distribution of all charged pion from a single "central" $100 \mathrm{Gev} / \mathrm{n} \mathrm{AU}+\mathrm{AU}$ event. The height of the bubble peak is about 3.5 times the background level. The bubble energy was $\approx 15 \%$ of the available energy. 


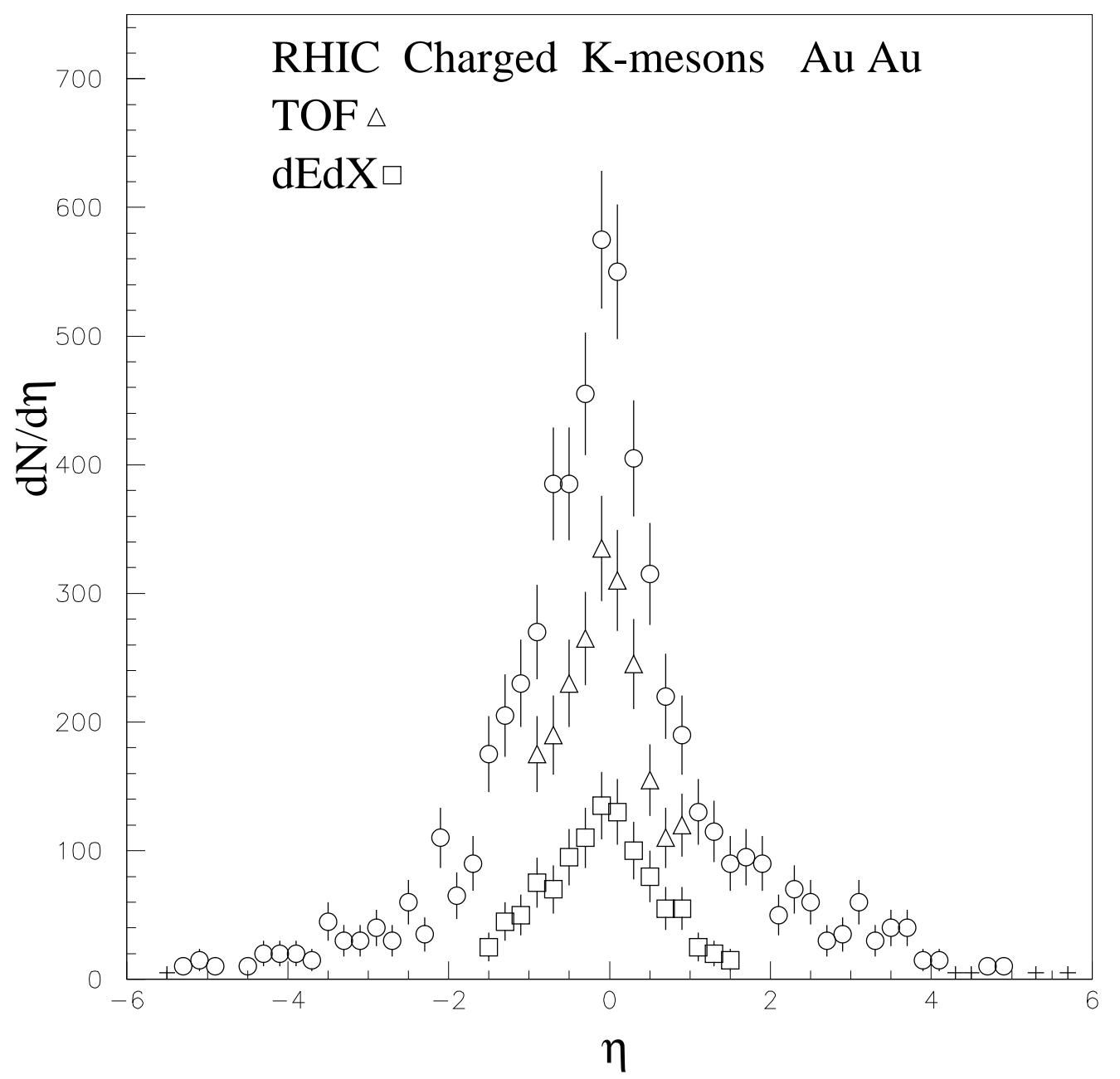

Figure 11: The pseudorapidity distribution for all charged K-mesons from a single "central" $100 \mathrm{Gev} / \mathrm{n}$ event, showing a peak about four times the background level. Both the TOF and DE/EX particle identifications show peaks 2-2.5 times the background level, but the TOF has much more statistics and detects more energetic particles. The bubble energy was $\approx$ $15 \%$ of the available energy. 


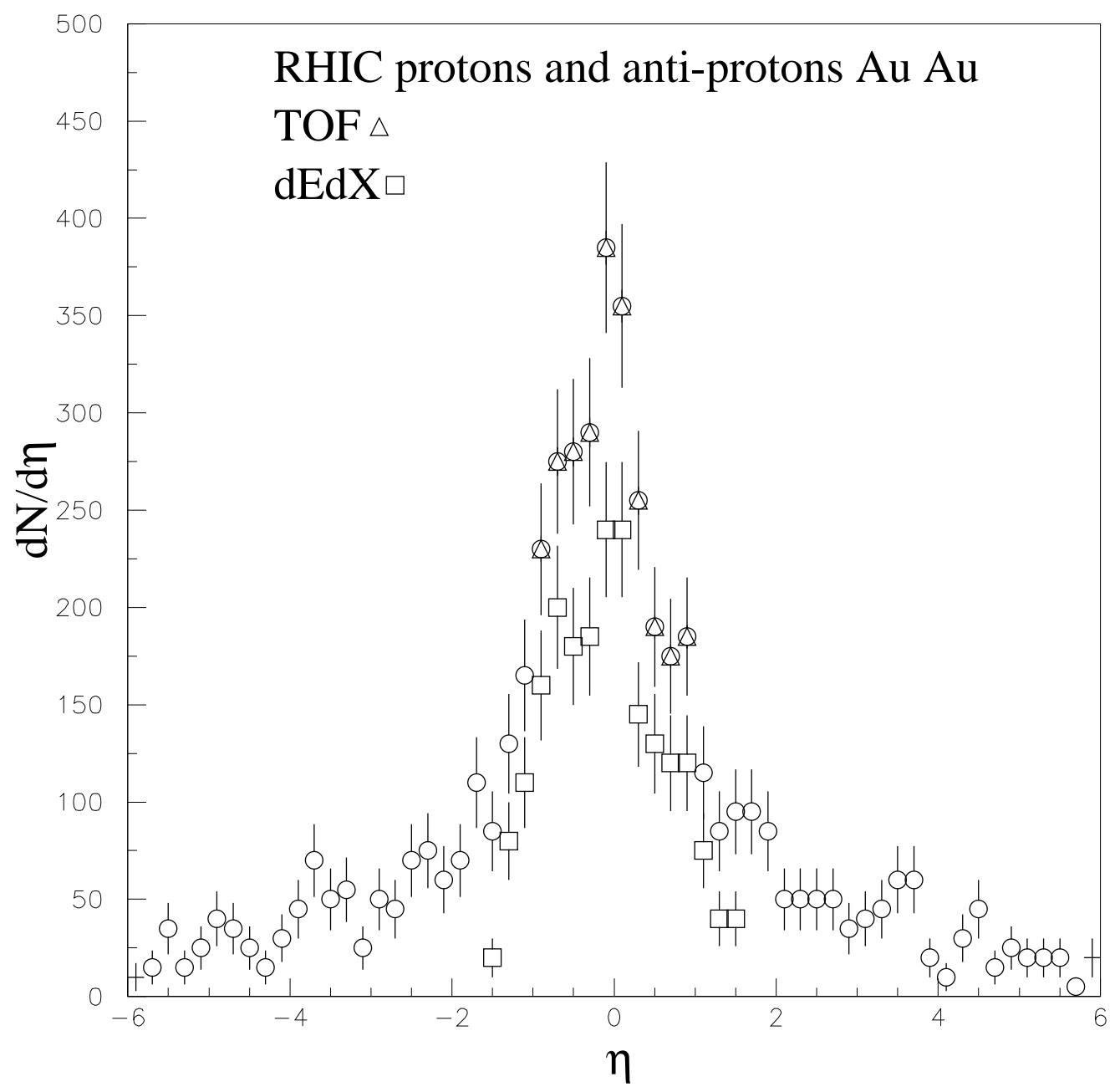

Figure 12: The pseudorapidity distribution for all protons and anti protons from a single "central" $100 \mathrm{Gev/n}$ event, showing a peak about four times the background level, but the TOF has much more statistics and detects more energetic particles. The bubble energy was $\approx 15 \%$ of the available energy. 


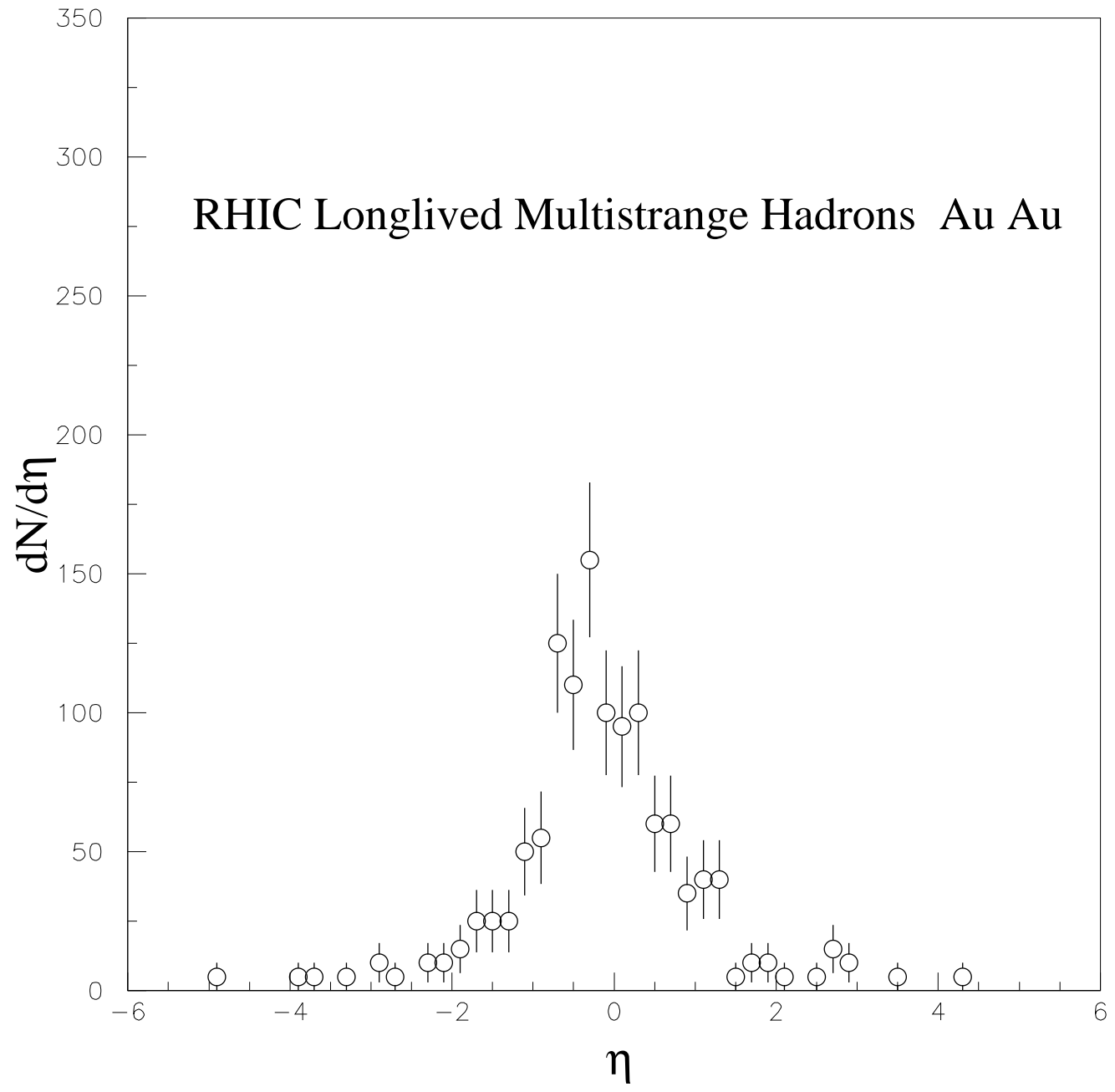

Figure 13: The pseudorapidity distribution for all long-lived Multistrange Hadrons $\left(\Xi, \Omega^{-}\right)$ from a single "central" $100 \mathrm{Gev} / \mathrm{n}$ event, showing a peak level about three times the background level. The bubble energy was $\approx 15 \%$ of the available energy. 


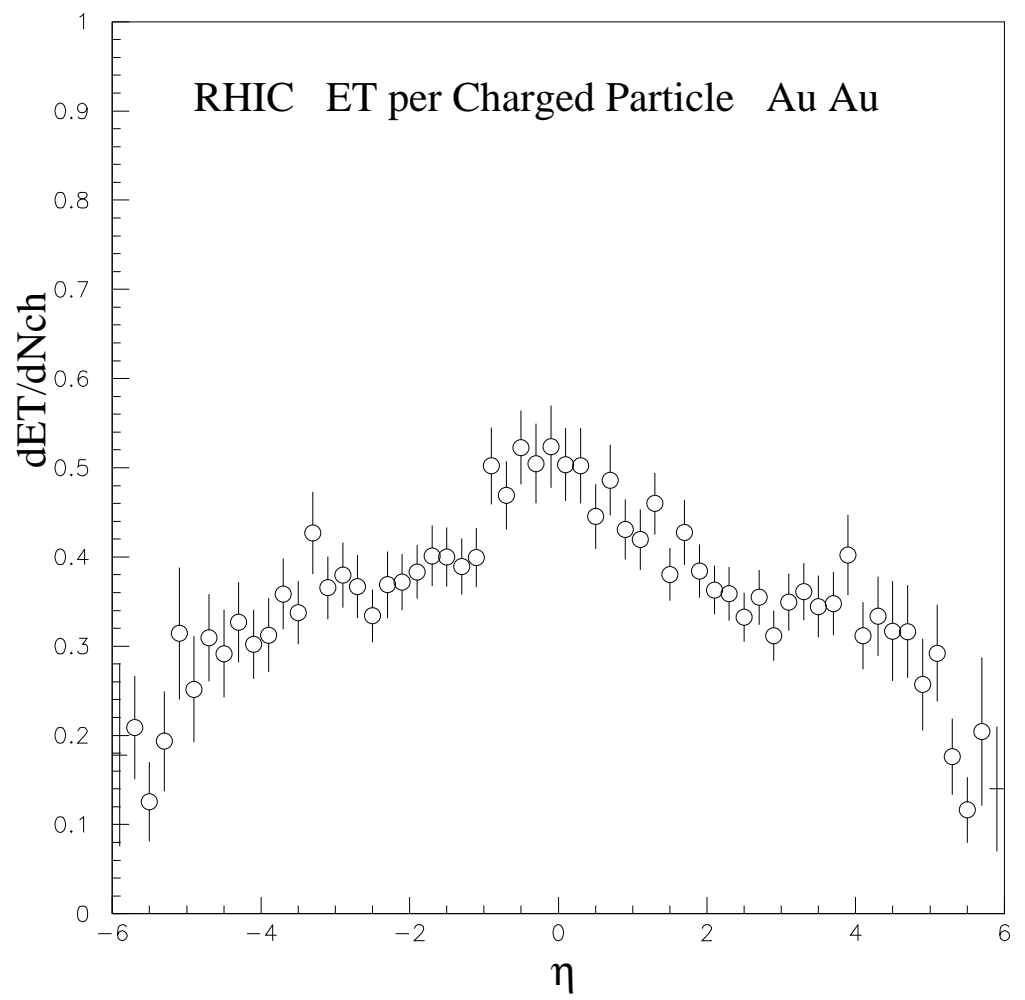

Figure 14: The pseudorapidity distribution of $\mathrm{dET} / \mathrm{dNch}$ (average transverse energy/per charged particle) for all charged particles from a single "central" $100 \mathrm{Gev} / \mathrm{n} \mathrm{Au}+\mathrm{Au}$ RHIC::EVENT (see Fig. 1). 


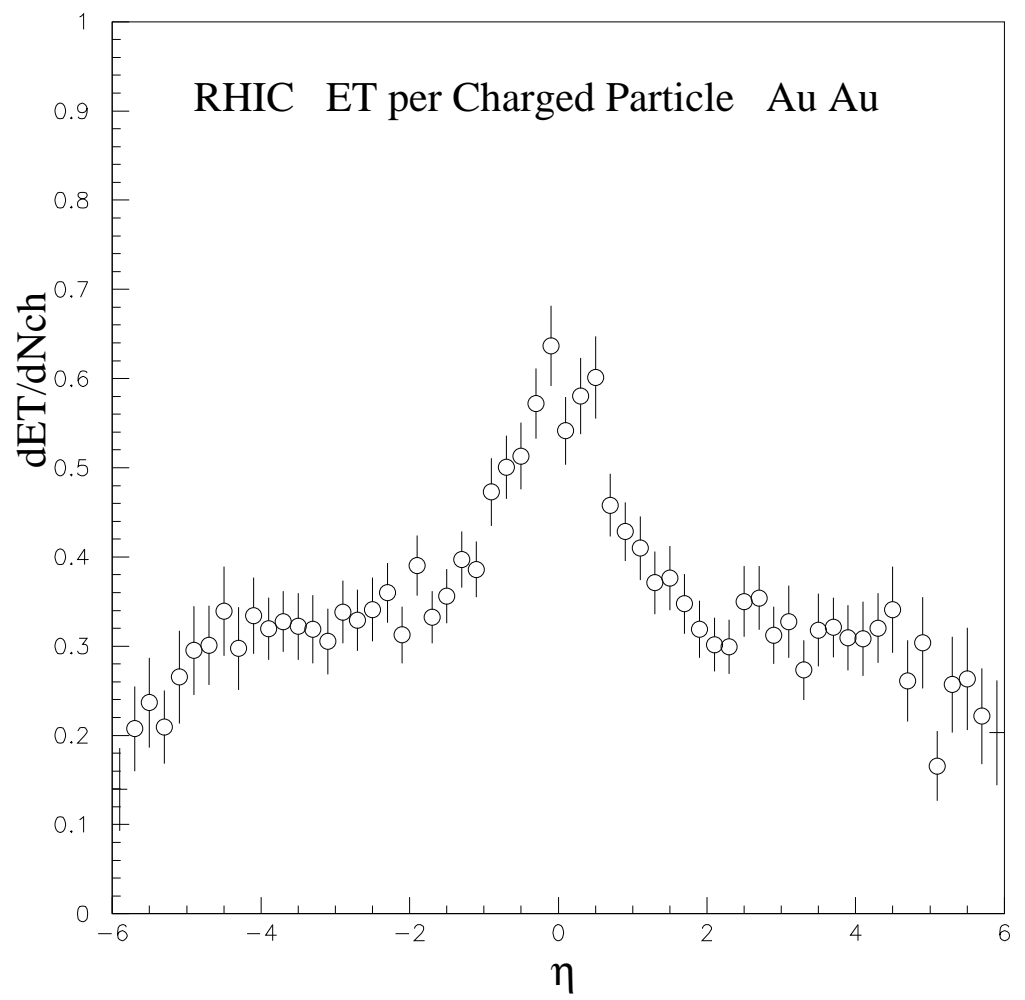

Figure 15: The pseudorapidity distribution of $\mathrm{dET} / \mathrm{dNch}$ (average transverse energy/per charged particle) for all charged particles from a single "central" $100 \mathrm{Gev} / \mathrm{n} \mathrm{Au}+\mathrm{Au}$ RHIC::PLASMA event (see Fig. 5). The bubble energy was $4.5 \%$ of the available energy. 


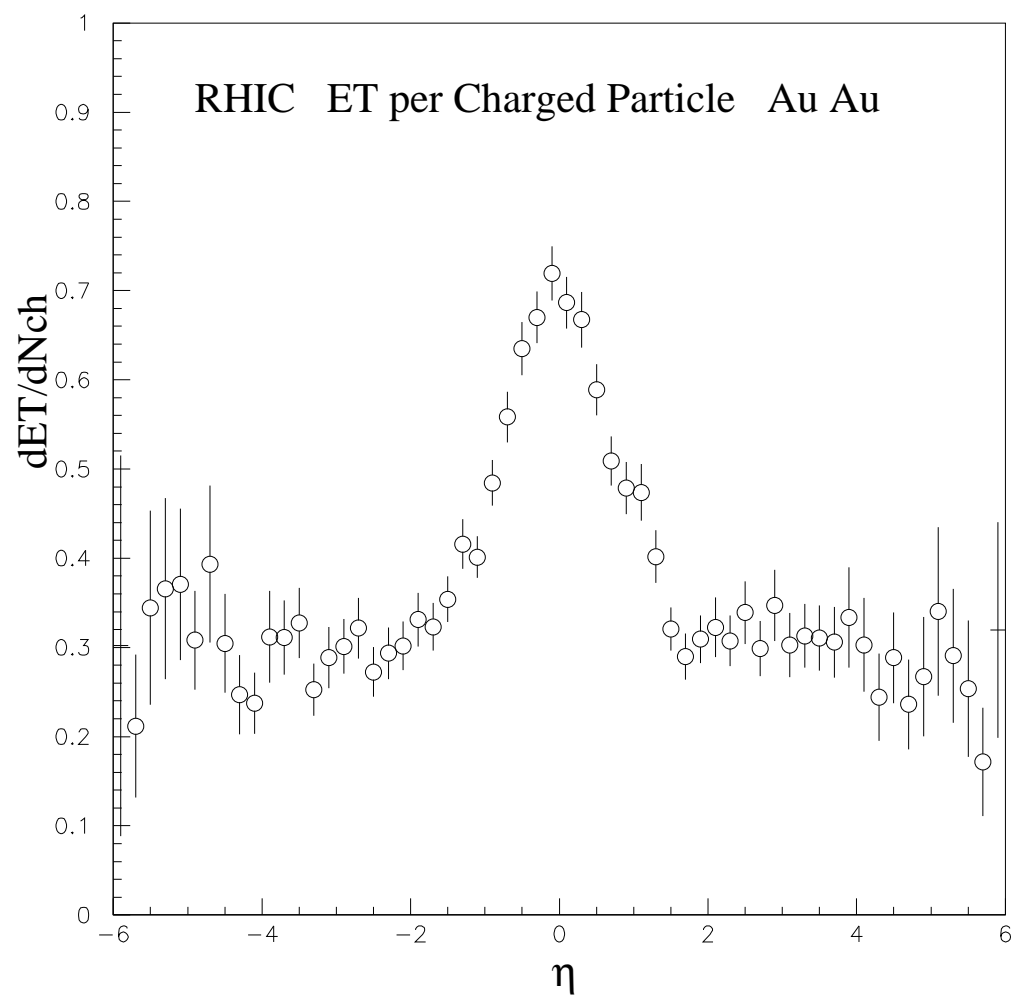

Figure 16: The pseudorapidity distribution of $\mathrm{dET} / \mathrm{dNch}$ (average transverse energy/per charged particle) for all charged particles from a single "central" $100 \mathrm{Gev} / \mathrm{n} \mathrm{Au}+\mathrm{Au}$ RHIC::PLASMA event (see Fig. 9). The bubble energy was $\approx 15 \%$ of the available energy. 


\section{REFERENCES}

[1] Quark Matter Formation and Heavy Ion Collisions: Proc. of the Biefeld Workshop, May 82. See papers by Jacobs and Satz, Baym, Kajantie, McLerran, Gyulassy, Knoll, Kapusta and others.

[2] Quark Matter conferences 1983, BNL; 1984 Helsinki, 1986 Asilomar, 1987 Nordkirschen, 1988 Lenox; 1990 Menton; 1991 Gatlinburg; 1993 Borlange, 1995 Monterey, 1996 Heidelberg, 1997 Tsukuba, 1998 Torino.

[3] Samios, N.P., RHIC Conceptual Design Report, (Brookhaven National Laboratory, BNL52195, (1989).

[4] L. Schroeder and S.J. Lindenbaum, Large Magnetic Spectrometers. Part II by S.J. Lindenbaum, RHIC Workshop: Experiments for a Relativistic Heavy Ion Collider, April 15-19, 1985, P.E. Haustein and C.L. Woody, Editors, pp. 227-252 (Brookhaven National Laboratory, Upton, New York, 1985), BNL51921.

[5] a) L. Schroeder and S.J. Lindenbaum, Large Magnetic Spectrometer. Lindenbaum, S.J., part II pp. 227-252 RHIC Workshop: Experiments for a Relativistic Heavy Ion Collider, April 15-19, 1985, P.E. Haustein and C.L. Woody, Editors, pp. 211-252 (Brookhaven National Laboratory, Upton, New York, 1985). (b) Lindenbaum, S.J. An Approximately 4p Tracking Magnetic Spectrometer for RHIC. Proc. of the Second Workshop on Experiments and Detectors for a Relativistic Heavy Ion Collider (RHIC), Lawrence Berkeley Laboratory, Berkeley, California, May 25-29, 1987, Editors, Hans Georg Ritter and Asher Shor, pp. 146-

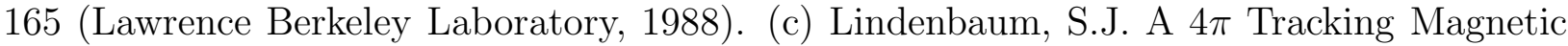
Spectrometer for RHIC. Proc. of the Third Workshop on Experiments and Detectors for a Relativistic Heavy Ion Collider (RHIC), Brookhaven National Laboratory, July 11-22, 1988, B. Shivakumar and P. Vincent, Editors, pp. 82-96 (Brookhaven National Laboratory, BNL 52185). (d) Lindenbaum, S.J. (Experimental Collaboration: G. Danby, S.E. Eiseman, A. Etkin, K.J. Foley, R.W. Hackenburg, R.S. Longacre, W.A. Love, T.W. Morris, E.D. Platner, A.C. Saulys, J.H. Van Dijk, S.J. Lindenbaum, C.S. Chan, M.A. Kramer, K. Zhao, N. Biswas, P. Kenney, J. Piekarz, D.L. Adams, S. Ahmad, B.E. Bonner, J.A.Buchanan, C.N. Chiou, J.M. Clement, M.D. Corcoran, T. Empl, H.E. Miettinen, G.S. Mutchler, J.B. Roberts, J. Skeens) A 4p Tracking TPC Magnetic Spectrometer for RHIC. Proc. of the Fourth Workshop on Experiments and Detectors for a Relativistic Heavy Ion Collider, Brookhaven National Laboratory, July 2-7, 1990, Editors: M. Fatyga and B. Moskowitz, pp. 169-206 (BNL, 1990).

[6] L. Van Hove. Z. Phys. C. Particles and Fields 21,93-98 (1983), Hadronization Model Quark-Gluon Plasma in Ultra-Relativistic Collisions CERN-TH 3924 (1984).

[7] L. Van Hove Nucl. Phys. A46 (1987).

[8] M. Gyulassy, H. Kajantie, Kurki-Suuno and L. McLerran, Nucl. Phys. B237 (1984) 477.

[9] F.E. Paige and S.D. Protopopescu, "ISAJET" A MONTE CARLO event generator program for pp and p-bar + p interactions; BNL-29777 (1991); BNL-31987, Sept. 1982 and modifications. 
[10] R.D. Field and R.P. Feynman, Nuci. Phys. B136, 1 (1978).

[11] T. Ludlam, et al., RHIC Workshop I, eds. P. Haustein and C.L. Woody (Brookhaven, April 85).

[12] X.N. Wang and M. Gyulassy. Phys. Rev. D 44 (1991) 3501; Phys. Rev. D 45 (1992) 844; Comp. Phys. Comm. 83 (1994) 307.

[13] B. Anderson, et al., Nucl. Phys. B 281, 289 (1987); Comp. Phys. Comm. 43, 387 (1987).

[14] T. Sjostrand, Comp. Phys. Comm 82, 74 (1994).

[15] M.Gyulassy, Nuclear Physics A 590 (19995) 431c-446c.

[16] A. Capella, U. Sukhatme, C. I. Tan and J. Tran Thanh Van, Phys. Rep. 236, 225 (1994).

[17] K. Werner, Phys. Rev. 282, 87 (1993).

[18] H. Sorge, H. Stocker, and W. Greiner, Nucl. Phys. A 498, 567c (1989).

[19] H. Sorge, H. Stocker and W. Greiner, Nucl. Phys. A 566, 663c (1994).

[20] K. Geiger and B Mueller, Nucl. Phys. B 369, 600 (1992).

[21] P. Koch, B. Müller and J. Rafelski, Phys. Rep. 142, 167 (1986).

[22] L. D. Landau, Izu. Akad. Nauk SSSR 17, 51 (1953).

[23] E. Schnedermann and U. Heinz, Phys. Rev. Lett. 69, 2908 (1992).

[24] JACEE Collaboration, Phys. Rev. Lett. 50, 2062 (1983).

[25] C.M.G. Lattes, Y. Fujimoto and S. Hasegawa, Phys. Rep. 65, 151 (1980); L.T. Baradzei et al., Nucl. Phys. B370, 365 (1992).

[26] K. Rajagopal and F. Wilczek, Nucl. Phys. B379, 395 (1993).

[27] We have chosen the 3 direction in isospin to be the neutral pions.

[28] T.J. Hallman, et al. (STAR Collaboration). The Physics and Detectors of the Relativistic Heavy Ion Collider (RHIC). Proc. XXXIst Rencontres de Moriond, Les Arcs. Savoie, France, March 23-30, 1996. Series: Moriond Particle Physics Meetings, '96 QCD and High Energy Hadronic Interactions, edited by J. Tran Thanh Van, published by Editions Frontieres, pp. 485-90 (1996).

[29] Klaus Geiger, Ron Longacre, and Dinesh K. Srivastava, VNI;Simulation of HighEnergy Particle Collisions in QCD, BNL-65755 (1998), Klaus Geiger and Ron Longacre Heavy Ion Physics 8, 41(1998).

[30] D. Kharzeev and R. Pisarski, hep-ph/9906401, January 16, 1999.

[31] D. Kharzeev, private communication.

[32] M. Gyulassy, RBRC Memo, 3/11/99. 
[33] R. Longacre, Parton structure through two particle correlations in Au-Au at RHIC.

Proc. RHIC Physics and Beyond: Kay Kay Gee Day, October 23, 1998, Brookhaven National Laboratory, Upton, NY (in press). 